\title{
Transmit Power Minimization and Base Station Planning for High-Speed Trains with Multiple Moving Relays in OFDMA Systems
}

\author{
Hakim Ghazzai, Member, IEEE, Taha Bouchoucha, Student Member, IEEE, Ahmad Alsharoa, Student \\ Member, IEEE, Elias Yaacoub, Senior Member, IEEE, Mohamed-Slim Alouini, Fellow, IEEE, and Tareq \\ Y. Al-Naffouri, Member, IEEE
}

\begin{abstract}
High-speed railway system equipped with moving relay stations placed on the middle of the ceiling of each train wagon is investigated. The users inside the train are served in two hops via the orthogonal frequency-division multiple access (OFDMA) technology. In this work, we first focus on minimizing the total downlink power consumption of the base station (BS) and the moving relays while respecting specific quality of service $(\mathrm{QoS})$ constraints. We first derive the optimal resource allocation solution in terms of OFDMA subcarriers and power allocation using the dual decomposition method. Then, we propose an efficient algorithm based on the Hungarian method in order to find a suboptimal but low complexity solution. Moreover, we propose an OFDMA planning solution for high-speed train by finding the maximal inter-BS distance given the required user data rates in order to perform seamless handover. Our simulation results illustrate the performance of the proposed resource allocation schemes in the case of the 3GPP Long Term Evolution-Advanced (LTE-A) and compare them with previously developed algorithms as well as with the direct transmission scenario. Our results also highlight the significant planning gain obtained thanks to the use of multiple relays instead of the conventional single relay scenario.
\end{abstract}

Index Terms-High-speed railway communication, moving relays, planning, resource allocation algorithm.

\section{INTRODUCTION}

With the growing technology of the smart phone, the passenger transport industry is witnessing a high demand to ensure Internet access, high-quality voice and/or mobile video broadcasting all the time inside the train. In addition

Copyright (c) 2015 IEEE. Personal use of this material is permitted. However, permission to use this material for any other purposes must be obtained from the IEEE by sending a request to pubs-permissions@iee.org.

A part of this work has been presented at the 12th International Symposium on Modeling and Optimization in Mobile, Ad Hoc, and Wireless Networks (WiOpt 2014), Hammamet, Tunisia, May 2014.

The work was made possible, in part, by NPRP grant \#6-001-2-001 from the Qatar National Research Fund (a member of Qatar Foundation). The statements made herein are solely the responsibility of the authors.

Hakim Ghazzai is with Qatar Mobility Innovations Center (QMIC), Qatar Science \& Technology Park, Doha, Qatar, e-mail: hakimg@qmic.com.

Taha Bouchoucha, Mohamed-Slim Alouini, and Tareq Al-Naffouri are with the Computer, Electrical and Mathematical Sciences and Engineering (CEMSE) Division, King Abdullah University of Science and Technology (KAUST), Thuwal, Makkah Province, Saudi Arabia, e-mails: \{taha.bouchoucha, slim.alouini, tareq.alnaffouri\}@kaust.edu.sa.

Ahmad Alsharoa is with Iowa State University (ISU), Ames, Iowa, USA, e-mail: alsharoa@iastate.edu.

Elias Yaacoub is with the Arab Open University (AOU), Beirut, Lebanon, e-mail: eliasy@ieee.org.

This work was done while Taha Bouchoucha was at KAUST. He is now in UC Davis, California, United States. to that, there is a pressing need to improve the coverage and handover process in high-speed communications to ensure safe information exchange between trains and terrestrial control centers such as information on the location of the train, its state, level crossings, permitted speed, etc. All these services require advanced technologies and techniques providing performance that meets the users' quality of service (QoS) [1]. Recently, high-speed railway networks have attracted an extensive research attention. The latest research progress, systems architecture, and future challenges and tradeoffs of this field are presented in [2]-[4]. In general, when the user equipment (UE) communicates directly with the base station (BS), it will suffer from a severe degradation of the QoS due to the path loss (PL) and shadowing effects as the wireless signal is traveling through the train. As an alternative solution to cope with this, the 3rd Generation Partnership Project (3GPP) has supported relay communications in the Long Term EvolutionAdvanced (LTE-A) release-10 standard [5]-[7].

In line with standardization effort [8], research studies using a mobile relay (MR) placed on top of the train are proposed. In [9], it is shown that MR-assisted transmission greatly outperforms transmission assisted by fixed relays as well as direct transmission for vehicular communications. Indeed, using MRs within the high-speed railway mobile communication helps in enhancing the cellular coverage and overcoming the moderate to high vehicle penetration loss (VPL) [10], [11]. VPL can be negligible by introducing two antennas connected together by a cable; outside and inside the vehicle. The outside antenna communicates with the BS, while the inside antenna communicates with the UEs inside the vehicle [12]-[14].

Many of the works dealing with high-speed railway communications are tackling the issue of resource allocation [15] and [16], particularly for orthogonal frequency division multiple access (OFDMA) systems either for direct links or with relayassistance [17]-[19]. Indeed, OFDMA system with relaying is considered as a fundamental solution for emerging cellular systems to ensure coverage and maintain connectivity [20], especially with MRs for vehicular communications. In [15], the authors investigated a utility-based power allocation problem at a BS communicating with a MR in high-speed railway wireless networks, while jointly considering the power allocation along the time and the packet allocation among services. In [16], a two-hop architecture where multiple BSs communicate with the train via a unique access point is studied. An optimal uplink power allocation policy which satisfies 
the delay constraint and minimizes the power consumption is provided. In [18], the author considered the constraint of bit error rate in the resource allocation process. A recent work is proposed in [19] investigating the resource allocation in an OFDMA relaying framework. The objective is to allocate power and subcarriers such that the capacity of the MR is maximized while guaranteeing that the sum capacity of local users inside the train is no less than a threshold. Another subcarrier allocation scheme with uniform power distribution has been investigated in [21]. The authors proposed an iterative resource allocation approach in order to maximize the total sum-rate of the UEs.

The cellular handover constitutes another major problem in high-speed railway systems. Communication interruption caused by handover failures can considerably degrade the users' experience. This issue has been investigated in literature by proposing various handover schemes. For instance, in [22], a power allocation scheme for a distributed antenna system is implemented to enhance the handover failure probability in a two-link architecture is proposed. Another soft handover scheme based on multi-radio access technology is proposed in [23]. The idea is to keep multiple heterogeneous network links of the relay at the same time and maintain the relay connection through one link during the handover process of the other link. The authors in [24] investigated the handover signaling process for multiple MRs in LTE networks while using control MR placed in the front of the train in addition to several general MRs to serve the users inside the train. The role of the control MR is to perform measurement and decide with the BS if a handover is needed or not. If the handover is required, the MRs perform a group handover procedure. It shown that this process helps in reducing the handover process time and the conservation of connectivity.

In this study, we contribute to the aforementioned previous works by investigating the resource allocation problem for two-hop high-speed railway communication assisted by multiple MRs in the generic case of OFDMA model from a power minimization perspective. As the number of users inside the train is limited, we formulate an optimization problem that aims to minimize the total transmit power consumption of the system, taking into account the power budget and respecting a certain QoS for each UE. More specifically, the data rate of each UE is restricted to be greater than a predefined data rate threshold. This leads to a more challenging problem compared to other investigated frameworks, limited to single relay scenario only, that consider the total sum-rate in either their objective functions or constraints [15], [16], [19]. In fact, meeting the target rate per UE while minimizing the transmit power consumption requires additional constraints that make the feasibility of the problem sensitive to various system parameters such as the target rate per UE, number of connected UEs per wagon, the train traveled distance, the power budget, etc. Furthermore, the proposed framework is more practical and fair since the sum-rate metric could be beneficial for some users with good channel quality at the expense of others.

A backward induction method is employed: first, we solve the optimization problem for the access link between each
MR and UEs, then, the problem is solved for the backhaul link between the BS and the MRs. Hence, the achieved rates of the backhaul link are automatically dependent on the performance of the access link. Two algorithms are proposed to solve the resource allocation problem. The first algorithm achieves optimal performance based on the dual decomposition method where we jointly optimize the power levels and the subcarriers allocation. A closed-form expression of the optimal power allocated to each receive antenna is also derived for fixed subcarrier allocation. The second method is based on a practical and low complexity algorithm that achieves sub-optimal solutions of the resource allocation problem. We compare the performance of both proposed approaches with those of a previously proposed iterative algorithm in addition to the direct transmission scenario. We show via simulations that our proposed methods maintain the system QoS for long-range distance compared to the other studies proposed in literature.

Next, based on the proposed power allocation scheme, we propose a planning method to determine the inter-BS distance in order to perform a seamless handover between neighbor BSs while serving the multiple MRs given the required data rate per UE. In this case, the handover is treated from a planning and physical layer perspective to identify a separation distance that maintains the desired QoS per UE. This is performed to avoid any communication interruption in the handover zones that are determined by introducing a tolerance parameter to the problem. Finally, a Monte Carlo simulation is performed in order to verify the effectiveness of the proposed planning scheme.

The rest of this paper is structured as follows. Section II presents the system model. In Section III, we formulate the resource allocation optimization problem and describe the dual decomposition and the proposed algorithm solutions, then, we propose the planning method for multiple BSs scenario. Simulation results are discussed in Section IV. Finally, Section V concludes the paper.

\section{System Model}

We consider a high-speed train consisting of $L$ wagons with $M$ UEs inside the train distributed randomly over the train wagons. The UEs are communicating with the BS, placed at a distance denoted by $d_{B}$, via MR stations located at the top of each wagon. Each MR station is composed of two antennas connected together by a cable and placed outside and inside the train wagon as shown in Fig.1.

The available spectrum is divided into $K$ subcarriers having a bandwidth denoted by $B_{\mathrm{SC}}$. In the first hop, which is also known as the backhaul link, the BS transmits different signals over different subcarriers to the outside antennas of the MRs. Consequently, in the second hop, which is known as the access link, the $i^{\text {th }}$ MR serves the $M_{i}$ UEs inside the $i^{\text {th }}$ train car such that $\sum_{i=1}^{L} M_{i}=M$. The transmitter $t$ (e.g., BS or relay depending on the considered link) power vector is denoted by $\boldsymbol{P}_{t}=\left[P_{t}^{(1)}, \ldots, P_{t}^{(k)}, \ldots, P_{t}^{(K)}\right]$. In our study, we propose to compare this high-speed train communication model using MRs with the direct high-speed train communication scenario where the users inside the train are communicating directly 


$$
P L_{B-R_{i}}^{(k)}\left(d_{B-R_{i}}\right)= \begin{cases}44.2+21.5 \log _{10}\left(d_{B-R_{i}}\right)+20 \log _{10}\left(f^{(k)}[\mathrm{GHz}] / 5\right), & 10 \mathrm{~m}<d_{B-R_{i}}<d_{B P} \\ 10.5+40.0 \log _{10}\left(d_{B-R_{i}}\right)-18.5 \log _{10}\left(h_{B}\right)- & d_{B P}<d_{B-R_{i}}<10 \mathrm{~km} \\ 18.5 \log _{10}\left(h_{T}\right)+1.5 \log _{10}\left(f^{(k)}[\mathrm{GHz}] / 5\right), & \end{cases}
$$

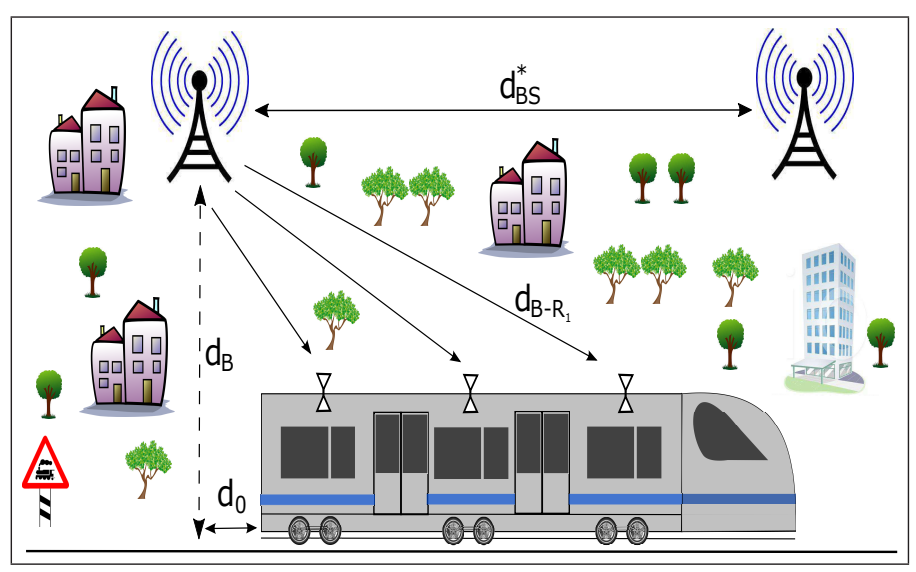

Fig. 1 High-Speed Train System Model.

with the BS. Notice that most of the studies about high-speed train communications are focusing on the downlink direction as the BS has limited power which will be shared among many UEs, unlike the uplink direction where each UE can use independently its power to perform the transmission to achieve the target data rate. Furthermore, thanks to MRs, the UEs inside wagons are subject to a short range communication that does not require sophisticated power optimization scheme in the uplink direction. In our study, we adopt the WINNER II channel model [25] to characterize the PL in order to benefit from its novel parametrization which allows the consideration of outdoor-to-indoor scenarios and the adaptation of the model to different environments.

\section{A. Path Loss Analysis for Relay Transmission (BS-Relay and Relay-User)}

The PL used in the backhaul link between the BS and the $i^{t h}$ MR (BS-MR link) over the $k^{t h}$ subcarrier under D1 line of sight (LoS) propagation scenario [25] is denoted by $P L_{B-R_{i}}^{(k)}$ and given in (1), where $d_{B-R_{i}}$ is the distance from the BS to the $i^{\text {th }}$ MR in meter, $f^{(k)}$ is the center frequency over the $k^{\text {th }}$ subcarrier in $\mathrm{Hz}, h_{B}$ and $h_{T}$ are the height of the $\mathrm{BS}$ and the train in meter, respectively, and $d_{B P}=4 h_{B} h_{T} f^{(k)} / c$ is the breaking point distance, where $c=3 \times 10^{8} \mathrm{~m} / \mathrm{s}$ is the speed of light in vacuum.

The PL used in the access link between the $i^{t h}$ MR and the $m^{\text {th }}$ UE (MR-UE link) belonging to the $i^{\text {th }}$ wagon over the $k^{\text {th }}$ subcarrier under A1 indoor LoS propagation scenario [25] is given by

$$
\begin{aligned}
P L_{R_{i}-U_{m}}^{(k)}\left(d_{R_{i}-U_{m}}\right)= & 46.4+18.7 \log _{10}\left(d_{R_{i}-U_{m}}\right)+ \\
& 20 \log _{10}\left(f^{(k)}[\mathrm{GHz}] / 5\right),
\end{aligned}
$$

where $d_{R_{i}-U_{m}}$ is the distance from the $i^{t h} \mathrm{MR}$ to the belonging $m^{t h}$ UE in meter.

\section{B. Path Loss Analysis for Direct Transmission (BS-User)}

In this section, the PL of the direct link between BS and UE without relay assistance under outdoor-indoor propagation scenario [25] is given by

$$
\begin{aligned}
P L_{B-U_{m}}^{(k)}\left(d_{U_{m}, \text { out }}, d_{U_{m}, \text { in }}\right)= & P L_{B-R}^{(k)}\left(d_{U_{m}, \text { out }}\right)+ \\
& P L_{R-U}^{(k)}\left(d_{U_{m}, \text { in }}\right)+P L_{\text {outer }},
\end{aligned}
$$

where $d_{U_{m} \text {,out }}$ and $d_{U_{m} \text {,in }}$ are the distance between the BS and the closest point of the train wall to the $m^{t h} \mathrm{UE}$ and the distance from train wall to the $m^{t h}$ UE in $m$, respectively. $P L_{\text {outer }}=W_{e}+W G_{e}(1-\cos (\theta))^{2}$ is the PL through the outer wall, where $W_{e}$ is the loss through the train wall for the perpendicular penetration, while $W G_{e}$ is the loss through the train wall for the parallel penetration, and $\theta$ is the angle between the normal of the train wall and the outgoing (incoming) ray.

All the aforementioned formulas are generalized for the frequency range $2-6 \mathrm{GHz}$ (for more details, refer to [25]).

\section{Channel Model}

The channel model in $[\mathrm{dB}]$ over the $k^{\text {th }}$ subcarrier is a function of the PL between transmitter $t$ and receiver $r$, shadowing, and fading as follows

$$
H_{t, r, \mathrm{~dB}}^{(k)}=-P L_{t, r}^{(k)}-\xi_{t, r}+20 \log _{10}\left(F_{t, r}^{(k)}\right),
$$

where $\xi_{t, r}$ is the log-normal shadowing between $t$ and $r$ with a standard deviation equals $\sigma$ and $F_{t, r}^{(k)}$ corresponds to the fast Rayleigh fading power between $t$ and $r$ such that $\mathbb{E}\left[|a|^{2}\right]=1$, where $a$ is a Gaussian random variable and $\mathbb{E}[$.$] is the expectation operator. In this case of fast Rayleigh$ fading, we consider that the channels between BS and UEs are constant during the channel de-correlated time $T_{d}$.

In (4), under the spatial log-normal shadowing assumption, the shadowing correlation is taken into account where the spatial correlation can be, indeed, used to measure how fast the local mean power evolves as the vehicle moves along a certain route [26]. Therefore, the shadowing correlation model for moving transmitter $t$ and receiver $r$ can be given by an exponential function [26], [27] as follows

$$
\Lambda_{\xi}\left(\Delta d_{t}, \Delta d_{r}\right)=\exp \left(-\frac{\Delta d_{t}+\Delta d_{r}}{l_{c}}\left(\log _{e} 2\right)\right),
$$

where $\exp ($.$) denotes the exponential function, \Delta d_{t}$ and $\Delta d_{r}$ represent the movements of the transmitter and receiver, respectively, and $l_{c}$ is the decorrelation length which depends on the environment (equal to 20 meters in the vehicular test environment [28]). Consequently, we can use (5) to determine the shadowing values as follows

$$
\begin{aligned}
\xi_{t, r}\left(d_{t}+\Delta d_{t}, d_{r}+\Delta d_{r}\right)= & \xi\left(d_{t}, d_{r}\right) \Lambda_{\xi}\left(\Delta d_{t}, \Delta d_{r}\right)+ \\
& \left(1-\xi\left(\Delta d_{t}, \Delta d_{r}\right)\right) \xi_{t, r}^{\text {new }},
\end{aligned}
$$


where the shadowing value at positions $\left(d_{t}+\Delta d_{t}, d_{r}+\right.$ $\Delta d_{r}$ ) has a component equal to the shadowing value at positions $\left(d_{t}, d_{r}\right)$, with the amount of similarity determined by $\Lambda_{\xi}\left(\Delta d_{t}, \Delta d_{r}\right)$, in addition to an independent shadowing component corresponding to the new location $\xi_{t, r}^{\text {new }}$. In our system model, we assume that $\Delta d_{t}=0$ since the BS has a fixed position.

Note that accurate channel estimation can be obtained for high-speed train communications thanks to the use of the predictor antennas concept [29]-[31]. A predictor antenna is placed on the top of the train followed by one or several separate receive antennas. The train is assumed to move through a stationary electromagnetic standing wave pattern which means that the BS creates a time-invariant pattern, and the train simply moves through it. Hence, receive antennas replace the predictor antenna and observe the same channel after a certain delay. With the knowledge of pilot signals, channel predictions can be performed at the receive antennas.

\section{Doppler Shift}

Doppler shift has been introduced as one of the important problems in high speed communications due to its negative impact on the system performance. For example, in OFDM systems where data is transmitted at a high speed over frequency selective channels, the longer duration of the OFDM symbol will be affected by the rapidly time-varying channel resulting from severe Doppler frequency shift which introduces intercarrier interference (ICI). Although the investigation of the Doppler effect is out of the scope of this paper, we present in this paragraph some techniques proposed to mitigate the Doppler effect for high-speed railway systems [32]-[34]. Note that the Doppler in the case of BS-MR links is easier to mitigate in the particular scenario of railway communications than other high-speed vehicular scenarios, since the speed and track of the train are known and predictable, in addition to the locations of the BSs along the rail track.

In [32], a sectorised antenna architecture is proposed to mitigate the Doppler effect in OFDM systems. It is shown that the signals received from different directional antennas are uncorrelated and after the approximated Doppler frequency compensation, the scheme reduces the ICI power by reducing the Doppler spread and mitigating the error floor under high mobility condition. In [33], Doppler spread compensation algorithms based on the dedicated radio environment map for railway are proposed. The radio environment map is employed as a spatial-temporal database consisting of the radio channel parameters along a given railway. With all these information that can be pre-determined and predicted offline, the REMbased Doppler spread compensation algorithms significantly improve the link performance of OFDM-based broadband mobile communication systems under various high-speed rail scenarios. An overview on the tracking of dynamic Doppler shift for LTE-based communication systems on high speed railway is presented in [34]. Algorithms dealing with the Large scale dynamic and fast time-varying Doppler shift for predictable route of a train are discussed.

The advanced techniques can be applied both at the BSs and MRs levels to reduce the impact of the Doppler shift.
The effect of the Doppler shift will be more significant on the backhaul link in the absence of MRs. Hence, the use of Doppler shift compensation algorithms with MRs represents a promising solution to reduce the negative impact of Doppler spread in OFDMA systems.

\section{Power Minimization-based Resource}

\section{Allocation and Base Station Planning}

The objective of this study is to ensure a seamless railway communication with minimum power consumption. Therefore, we proceed by solving an optimization problem aiming to find the optimal resource allocation in terms of subcarriers and power levels at the BS and MRs in order to minimize the total transmit power consumption while respecting the UE QoS. In other words, the optimization problem purpose is to serve all users inside the $L$ wagons of the train with minimum power consumption. In Section III-A and Section III-B, we formulate the optimization problem and propose two approaches to solve it, respectively. The first one is optimal but complex, and the second one is sub-optimal but with low complexity. The nature of the system implies that the problem could be infeasible and hence, impossible to solve due to limited BS power budget. Hence, we propose, in Section III-C, a BS planning approach to cope with this issue. The aim is to determine the BSs' maximum range after which the QoS will be deteriorated and there is no more possibility to serve all MRs and hence, all UEs, with the same BS.

\section{A. Problem formulation}

A receiver $r$ (i.e., a MR or UE depending on the link) is considered successfully served if its data rate $R_{r} \geq R_{\mathrm{th}}^{r}$ where $R_{r}$ is given by

$$
R_{r}\left(\boldsymbol{P}_{t}\right)=\sum_{k=1}^{K} \epsilon(k, r) B_{\mathrm{SC}} \log _{2}\left(1+\gamma_{t, r}^{(k)}\left(P_{t}^{(k)}\right)\right),
$$

where $\epsilon$ is either a matrix of size $K \times L$ such that $K \geq L$ if we consider the backhaul link or $K \times M_{i}$ such that $K \geq M_{i}$ if we consider the access link inside wagon $i$. Binary variables $\epsilon(k, r)$ refer to the status of the $k^{t h}$ subcarrier whether it is allocated to receiver $r$ or not and it is given as follows

$\epsilon(k, r)= \begin{cases}1, & \text { if } k^{\text {th }} \text { subcarrier is allocated to receiver } r, \\ 0, & \text { otherwise. }\end{cases}$

$R_{\mathrm{th}}^{r}$ corresponds to the data rate threshold of the receiver $r$. In (7), $\gamma_{t, r}^{(k)}$ is the received signal-to-interference-plus-noise ratio at the receiver over the $k^{\text {th }}$ subcarrier and is given as follows

$$
\gamma_{t, r}^{(k)}\left(P_{t}^{(k)}\right)=\frac{P_{t}^{(k)} H_{t, r}^{(k)} G_{t} G_{r}}{I+N_{0} B_{\mathrm{SC}}},
$$

where $G_{t}$ and $G_{r}$ are the transmitter and receiver antenna gains, respectively. $I$ and $N_{0}$ are the average co-channel interference caused by other BSs and/or other MRs and the 
noise variance, respectively. Thus, the optimization problem for each backhaul or access link can be formulated as

$$
\underset{\boldsymbol{\epsilon}, \boldsymbol{P}_{t} \geq 0}{\operatorname{minimize}} \sum_{k=1}^{K} P_{t}^{(k)} \sum_{r=1}^{M_{i}, L} \epsilon(k, r)
$$

subject to:

(C1: Power budget constraint at the transmitter $t$ ):

$$
\sum_{k=1}^{K} P_{t}^{(k)} \sum_{r=1}^{M_{i}, L} \epsilon(k, r) \leq \bar{P}_{t},
$$

(C2: Receiver $r$ rate constraint):

$R_{r}\left(\boldsymbol{P}_{t}\right) \geq R_{\text {th }}^{r}, \quad \forall r$,

(C3: Subcarrier selection constraints):

$$
\sum_{r=1}^{M_{i}, L} \epsilon(k, r) \leq 1, \forall k \text {, and } \sum_{k=1}^{K} \epsilon(k, r) \leq K, \forall r,
$$

where $\bar{P}_{t}$ is the peak power at the transmitter $t$.

Constraint (12) ensures that the QoS of the receiver $r$ is satisfied, while constraint (13) indicates that each subcarrier can be allocated to only one UE and, at maximum, one UE can be served by $K$ subcarriers. Note that constraint (12) is written as $R_{U_{m}}\left(\boldsymbol{P}_{R_{i}}\right) \geq R_{\mathrm{th}}^{U_{m}}, \quad \forall m=1, \cdots, M_{i}$, for the access link inside wagon $i$, and as $R_{R_{i}}\left(\boldsymbol{P}_{B}\right) \geq R_{\mathrm{th}}^{R_{i}}, \quad \forall i=$ $1, \cdots, L$, for the backhaul link between the BS and MRs. The challenge now is to enhance the performance of the system by optimizing the power levels simultaneously with the subcarriers allocation.

\section{B. Problem Solution}

In this section, we propose two solutions in order to solve the optimization problem formulated in (10)-(13): the dual decomposition method that achieves the optimal solution but requires a high computational complexity, and a practical and low complexity algorithm that reaches a suboptimal solution. The loss in terms of performance and gain in terms of complexity are discussed in Section IV. In order to solve the resource allocation problem for high-speed train communication, we proceed as follows: First, we focus on the access link to find the optimal resource allocation at each MR station. Depending on the number of UEs $M_{i}$ per wagon $i$ and the corresponding target data rate $R_{\mathrm{th}}^{U}$, we can compute the target data rate per each MR station denoted by $R_{\mathrm{th}}^{R_{i}}, \forall i=1, \cdots, L$, which is equivalent to the sum of the target rates of the UEs it is serving. Once $R_{\mathrm{th}}^{R_{i}}$ becomes known for each MR $i$, we can then focus on the backhaul link and find the optimal resource allocation at the BS. By doing this, in the backhaul link, we assign few resources to MRs that have low $R_{\mathrm{th}}^{R_{i}}$ and assign more resources to MRs that have high $R_{\mathrm{th}}^{R_{i}}$.

Note that in extreme scenarios, e.g., high rate threshold per user, train at the cell edge, and/or limited power budget, the problem (10)-(13) becomes infeasible as the power budget becomes insufficient to serve all UEs. For instance, for a given subcarrier allocation and using (11) and (12), the problem is infeasible if:

$$
\sum_{k=1}^{K}\left(2^{\frac{R_{\mathrm{th}}^{r}}{\overline{B S C}_{\mathrm{SC}}}}-1\right) \frac{I+N_{0} B_{\mathrm{SC}}}{H_{t, r}^{(k)} G_{t} G_{r}} \geq \bar{P}_{t},
$$

In this case, the problem can be relaxed by iteratively eliminating some users at the farthest MRs and hence, reducing the target data rate per MR, till the problem becomes feasible. By this way, the problem aims to serve the maximum number of users given the available power budget. However, this approach could have a negative impact on the global QoS of the system. Therefore, in Section III-C, we focus on determining the best locations of neighbor BSs to avoid such QoS deterioration.

1) Dual Decomposition Method and Optimal Solution: The problem in (10)-(13) satisfies the dual time sharing condition investigated in [35]. Thus, the duality gap of this non-convex resource allocation problem in OFDMA multicarrier system is negligible as the number of subcarriers is sufficiently large compared to the number of users. Therefore, we solve it by exploiting its dual problem that is expressed by

$$
\begin{aligned}
& \underset{\boldsymbol{\lambda}}{\operatorname{maximize}} g(\boldsymbol{\lambda}), \\
& \text { subject to: }(13),
\end{aligned}
$$

where $\boldsymbol{\lambda}=\left[\lambda_{0}, \lambda_{1}, \ldots, \lambda_{M_{i}}, \ldots, \lambda_{M_{i}+L}\right]$ is the vector that contains the Lagrange multipliers associated to constraints (11) and (12). The dual function $g(\boldsymbol{\lambda})$ is given as follows

$$
g(\boldsymbol{\lambda}) \triangleq \underset{\epsilon, \boldsymbol{P}_{t} \geq 0}{\operatorname{minimize}} \quad \mathcal{L}(\boldsymbol{\lambda}),
$$

subject to: (13).

where $\mathcal{L}(\boldsymbol{\lambda})$ is the Lagrangian expressed as

$$
\begin{aligned}
\mathcal{L} & =\sum_{k=1}^{K} \sum_{r=1}^{M_{i}, L} P_{t}^{(k)} \epsilon(k, r)+\lambda_{0}\left[\sum_{k=1}^{K} \sum_{r=1}^{M_{i}, L} P_{t}^{(k)} \epsilon(k, r)-\bar{P}_{t}\right] \\
& +\sum_{r=1}^{M_{i}, L} \lambda_{r}\left[R_{\mathrm{th}}^{r}-\sum_{k=1}^{K} \epsilon(k, r) B_{\mathrm{SC}} \log _{2}\left(1+\gamma_{t, r}^{(k)}\left(P_{t}^{(k)}\right)\right)\right] .
\end{aligned}
$$

Thus, the dual problem in (16) can be rewritten as follows

$$
\begin{aligned}
& g(\boldsymbol{\lambda})=\underset{\boldsymbol{\epsilon}, \boldsymbol{P}_{t} \geq 0}{\operatorname{minimize}} \sum_{k=1}^{K} \sum_{r=1}^{M_{i}, L} \epsilon(k, r) \mathcal{D}\left(P_{t}^{(k)}\right)-\lambda_{0} \bar{P}_{t} \\
& +\sum_{r=1}^{M_{i}, L} \lambda_{r} R_{\mathrm{th}}^{r}, \\
& \text { subject to: }
\end{aligned}
$$

where

$\mathcal{D}\left(P_{t}^{(k)}\right)=\left(1+\lambda_{0}\right) P_{t}^{(k)}-\lambda_{r} B_{\mathrm{SC}} \log _{2}\left(1+\gamma_{t, r}^{(k)}\left(P_{t}^{(k)}\right)\right)$.

The steps to solve the dual problem are given as follows:

- Step 1: Initialize the Lagrange multipliers values $\lambda$.

- Step 2: Find the optimal value of $P_{t}^{(k)}$ for each value of $k$ by solving the following problem

$$
\underset{P_{t}^{(k)} \geq 0}{\operatorname{minimize}} \mathcal{D}\left(P_{t}^{(k)}\right) .
$$

The corresponding optimal power $\left(P_{t}^{(k)}\right)^{*}$ is given by

$$
\left(P_{t}^{(k)}\right)^{*}=\left(\frac{\lambda_{r} B_{\mathrm{SC}}}{\left(\log _{e} 2\right)\left(1+\lambda_{0}\right)}-\frac{I+N_{0} B_{\mathrm{SC}}}{H_{t, r}^{(k)} G_{t} G_{r}}\right)^{+},
$$


where $(x)^{+}$corresponds to the maximum between $x$ and zero, and $\lambda_{r_{k}}$ is the Lagrange multiplier associated to receiver $r$ served by subcarrier $k$ since from constraint (13), each subcarrier serves only one receiver. In order to solve this analytical expression based on $\boldsymbol{\lambda}$, we can employ the subgradient method or other heuristic approaches to find the optimal Lagrangian multipliers of this problem [36].

- Step 3: Substitute the optimal power levels derived in (20) into (18). Thus, the dual problem becomes

$$
\begin{aligned}
& g(\boldsymbol{\lambda})=\underset{\epsilon, \boldsymbol{P}_{\boldsymbol{t}} \geq 0}{\operatorname{minimize}} \sum_{k=1}^{K} \sum_{r=1}^{M_{i}, L} \epsilon(k, r) \mathcal{D}\left(\left(P_{t}^{(k)}\right)^{*}\right) \\
& -\lambda_{0} \bar{P}_{t}+\sum_{r=1}^{M_{i}, L} \lambda_{r} R_{\mathrm{th}}^{r},
\end{aligned}
$$

subject to: (13),

It can be shown that (21) is a linear assignment problem with respect to $\epsilon(k, r)$ and can be solved efficiently by using standard integer programming [37]. The solution obtained by the dual method is an asymptotically optimal solution [35].

- Step 4: After finding the optimal solutions $\left(P_{t}^{(k)}\right)^{*}$ and $\epsilon(k, r)^{*}$ corresponding to the initialized Lagrange multipliers in Step 1, we employ the subgradient method to find their optimal values and thus the optimal solution of the problem [36]. Hence, to obtain the solution, we can start with any initial values for the Lagrange multipliers and evaluate the optimal solutions (i.e., $\left(P_{t}^{(k)}\right)^{*}$ and $\left.\epsilon(k, r)^{*}\right)$. We then update the Lagrange multipliers at the next iteration $(i+1)$ as follows

$$
\begin{gathered}
\lambda_{0}^{(i+1)}=\lambda_{0}^{(i)}-\delta^{i}\left(\bar{P}_{t}-\sum_{k=1}^{K} P_{t}^{(k)} \sum_{r=1}^{M_{i}, L} \epsilon(k, r)\right), \\
\lambda_{r}^{(i+1)}=\lambda_{r}^{(i)}+\varpi^{i}\left(R_{r}\left(\boldsymbol{P}_{t}\right)-R_{\mathrm{th}}^{r}\right), \quad \forall r,
\end{gathered}
$$

where $\delta^{i}$ and $\varpi^{i}$ are the updated step size according to the nonsummable diminishing step length policy (see [36] for more details). The updated values of the optimal solution and the Lagrange multipliers are repeated until convergence.

2) Low Complexity Algorithm and Suboptimal Solution:

The steps of the proposed approach for the resource allocation algorithm can be described as follows:

- Step 1: Simplify the optimization problem by distributing the peak power of the $i^{\text {th }}$ MR uniformly over its belonging subcarriers (i.e., $\bar{P}_{R_{i}}^{(k)}=\frac{\bar{P}_{R_{i}}}{K}$ ) where $\bar{P}_{R_{i}}^{(k)}$ is the peak transmit power at the $k^{\text {th }}$ subcarrier of the $i^{\text {th }}$ MR. This means that constraint (11) becomes as follows

$$
P_{R_{i}}^{(k)} \leq \bar{P}_{R_{i}}^{(k)}, \forall k=1, \ldots, K .
$$

Thus, the solution of the optimization problem becomes as follows:

$$
P_{R_{i}}^{(k)}= \begin{cases}\frac{A_{\mathrm{th}}}{H_{R_{i}, U_{m}}^{(k)}}, & \text { if } H_{R_{i}, U_{m}}^{(k)} \geq \frac{A_{\mathrm{th}}}{\bar{P}_{R_{i}}^{(k)}}, \\ 0, & \text { otherwise, }\end{cases}
$$

where $A_{\mathrm{th}}=\left(2^{\frac{R_{\mathrm{th}}^{U}}{\mathrm{BSC}_{\mathrm{SC}}}}-1\right)\left(I+N_{0} B_{\mathrm{SC}}\right)$. The obtained solution derived in (25) means that the UE $m$ served by the $i^{\text {th }}$ MR over subcarrier $k$ can achieve its data rate only if the corresponding channel is relatively good.

- Step 2: Compute $P_{R_{i}}^{(k)}$ for all possible (subcarrier, UE) combinations (i.e., $K \times M_{i}$ possibilities) inside the $i^{t h}$ wagon.

- Step 3: Employ a combinatorial optimization approach such as the Hungarian algorithm [38] to find the best (subcarrier, UE) combinations that maximizes the total number of served UEs with minimum power consumption inside the $i^{\text {th }}$ wagon. However, in some cases due to the modification of constraint (13), this method is not enough to serve the maximum number of UEs. Indeed, after allocating the subcarriers, some UEs may not achieve the required rate because of the power limitation as expressed in (24).

- Step 4: If for the wagon $i$, the number of served UEs is less than $M_{i}$, redistribute uniformly the remaining power over the remaining subcarriers $\left(\bar{P}_{R_{i}}=\bar{P}_{R_{i}}-\sum_{k=1}^{K} P_{R_{i}}^{(k)}\right)$ and repeat Steps 1 to 3 for the non-served UEs in wagon $i$. In fact, the peak power per subcarrier may increase comparing to Step 1.

- Step 5: Repeat Step 4 until serving all UEs or the remaining power per subcarrier is not enough to achieve the UE target data rate. In the latter case, try to serve at least one UE among of the remaining UEs by allocating the total remaining power to the UE having the best channel gain.

Note that the proposed approach is applied for the $L$ wagons simultaneously. At the end of the algorithm, $R_{\mathrm{th}}^{R_{i}}, \forall i=$ $1, \cdots, L$, becomes known and the BS applies the same approach but for the backhaul link. However, if at least one of the MRs is not served, we eliminate some UEs in the corresponding wagon in order to decrease the target data rate of the MR and thus try to serve the maximum number of UEs in that wagon. Details of the proposed algorithm are summarized in Algorithm 1 by replacing $Q$ and $t$ either by $M_{i}$ and $R_{i}$ for the access link or by $L$ and $B$ for the backhaul link, respectively. Once we find the allocation matrix $\epsilon^{*}$ and the corresponding total power consumption using our proposed approach, we compare the obtained results with the optimal analytical solution obtained using the dual decomposition method. As it will be shown in the simulation results section, the proposed low complexity approach achieves very close performance comparing with the optimal solution.

\section{Base Station Planning for High-Speed Trains}

In the case of multiple BSs separated by a distance $d_{\mathrm{BS}}$ along a high-speed railway, the QoS may not be maintained to the desired level while the train crosses the different cells due to possible handover failure as illustrates Fig. 2 where we consider a scenario where $3 \mathrm{BSs}$ are placed next to the railroad and separated by a distance of 3800 meters. We assume that the train is moving from the cell of BS-1 to the cell of BS3. Fig. 2 plots the total consumed power of the BS versus 


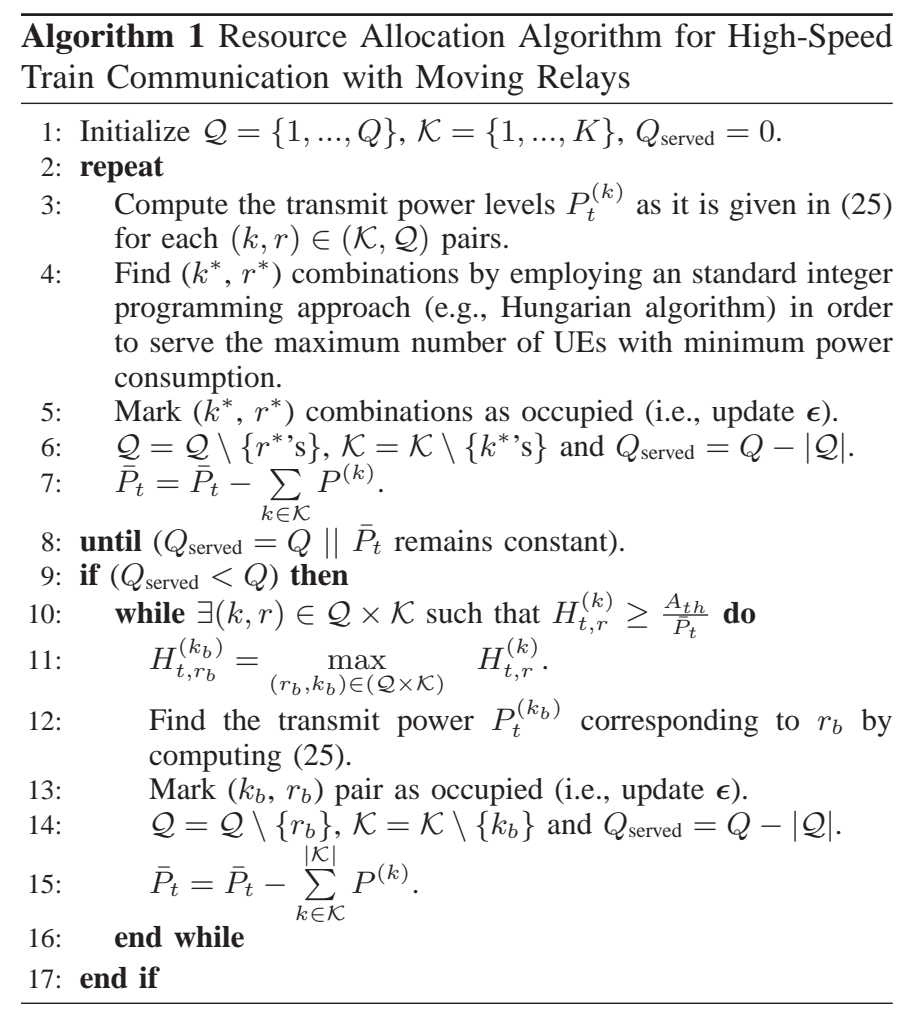

the train traveled distance and shows that the total consumed power exceeds the available power resources assumed to be $46 \mathrm{dBm}$ for a macrocell $\mathrm{BS}$ (the horizontal red line) in the handover zones. In other words, the optimization problem is infeasible in these regions. Therefore, based on the closedform expression of the allocated transmit power given in (20), we aim to optimize the choice of the inter-BS distance $d_{\mathrm{BS}}$ to avoid communication interruption and ensure a seamless handover between the different BSs.

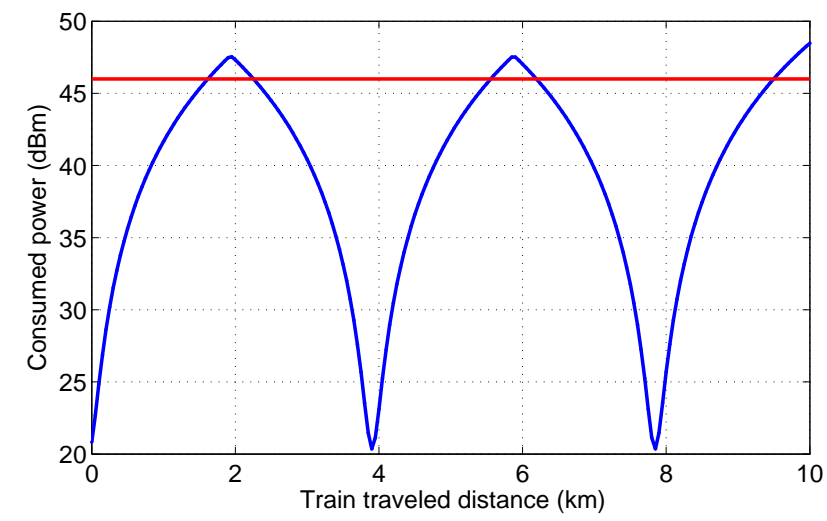

Fig. 2 Total consumed power in a high-speed railway with unoptimized planning (blue), total power available: $46 \mathrm{dBm}$ (red).

1) Proposed Planning Method: In this section, we provide a study of the inter-BS distance choice. We look for the maximum distance which maintains the desired QoS. We start by solving the following equation which ensures the consumption of the total power available by one BS to serve the $L$ MRs.

$$
\sum_{k=1}^{L} P_{t}^{(k)}\left(d_{B-R_{1}}\right)=\tau \bar{P}_{t}
$$

where $P_{t}^{(k)}\left(d_{B-R_{1}}\right)$ is the optimal power assigned to the $k^{t h}$ MR and $0<\tau \leq 1$ is a coefficient controlling the overlap in the handover zones. $\tau=1$ is the case of non-overlap and $\tau<1$ is the case where an overlap between the BSs' coverage areas is allowed in order to provide some milliseconds to perform the handover process and ensure a seamless communication. $P_{t}^{(k)}\left(d_{B-R_{1}}\right)$ is the solution of the power minimization-based resource allocation problem studied in Section III-A and it is given by the expression (20) which depends on the distance $d_{B-R_{1}}$ between the $\mathrm{BS}$ and the MR located at the first wagon of the train. Note that the distance $d_{B-R_{i}}$ between the BS and the MR located at the $i^{\text {th }}$ wagon, where $i=2, \ldots, L$ can be written in function of $d_{B-R_{1}}$ as follows

$$
d_{B-R_{i}}=\sqrt{d_{B}^{2}+\left(\sqrt{d_{B-R_{1}}^{2}-d_{B}^{2}}-(i-1) L_{w}\right)^{2}},
$$

where $L_{w}$ is the length of the wagon of the train.

The problem (26) corresponds to a non-linear equation that can be numerically solved using the Newton method [39]. Once the solution $d_{B-R_{1}}^{*}$ is obtained, we can directly find the planning distance using the following relation:

$$
d_{\mathrm{BS}}^{*}=2 \sqrt{\left(d_{B-R_{1}}^{*}\right)^{2}-d_{B}^{2}} .
$$

Note that in the proposed planning method, we are considering PL only in the channel model since we are determining the distance for average channel statistics. However, the channel model in the Monte-Carlo simulations presented in the following Section III-C2 includes both shadowing and fast fading to verify that the planning methods outcome is able indeed to meet the target performance requirements for instantaneous channel realizations.

Generally, for traditional cellular networks, two approaches could be considered to determine the BS locations: a worstcase scenario-based approach assuming maximum data rate and load per each BS and may lead to an excessive number of BSs, and a load-predictive approach which is more realistic [40]. In the case of railway communications, it is easier to predict the system requirement and, the worst-case scenario becomes somehow very close to the predictive case since the users' location and distribution in addition to the train trajectory are known. Also, it is known that the number of users cannot exceed the train capacity. Hence, the only requirements will be the target rate to provide to all users and the number of wagons per train. Consequently, mobile operators need to perform their planning for long-term periods such that major adjustments, like new BSs placement, are not required to ensure seamless communications.

2) Performance Evaluation Method: After determining the optimal distance for a given target data rate per UE and a given tolerance parameter $\tau$, we run a Monte Carlo simulation in order to investigate the impact of the proposed planning approach on the downlink direction. Thus, we measure the efficiency of the proposed scheme in realistic scenarios. In 
each realization, we consider $L$ MRs in a train which is moving in the railway line covered by three BSs. Then, we verify whether a relay $r$ is served successfully or not by comparing its achieved data rate, denoted by $R_{u}$, to the target data rate threshold, denoted by $R_{\mathrm{th}}$. The objective is to determine the average outage rate which has to be extremely low mainly in the handover zones determined thanks to the proposed planning method. In order to compute the data rates, we employ the channel gain given by (4) where, we take into account the propagation loss, the shadowing and the fading effects.

\section{Simulation Results}

In this section, we investigate and discuss the performance achieved by the proposed resource allocation schemes presented in Section III-B while comparing them to multiple approaches used in Literature. Then, we analyze their computational complexity in terms of total number of operations and CPU running time. Finally, we provide some numerical results of the performance obtained using the proposed planning approach.

\section{A. Simulation Parameters}

We consider a high-speed train scenario where the train is constituted of $L=10$ wagons, unless otherwise stated, of 10 $\mathrm{m}$ length, $5 \mathrm{~m}$ width, and $2.5 \mathrm{~m}$ height. We adopt the LTE-A transmission mode as an example of an OFDMA system. In LTE, the available spectrum is divided into resource blocks (RBs) consisting of 12 adjacent highly correlated subcarriers [41]. Each RB has a bandwidth of $B_{\mathrm{RB}}=180 \mathrm{kHz}$ while each subcarrier has a bandwidth of $B_{\text {sub }}=15 \mathrm{kHz}$ [42]. In our simulations, we consider an orthogonal LTE transmission where the total bandwidth of $B_{T}=20 \mathrm{MHz}$ is subdivided into three blocks. The first block of $10 \mathrm{MHz}$ (equivalent to 50 orthogonal RBs) is owned by the eNodeB (eNB), while the other blocks, each of $5 \mathrm{MHz}$ (equivalent to 25 orthogonal RBs), are owned by two MRs $i$ and $i+1, \forall i=1, \cdots, L-1$ [42], [43]. Hence, the same frequency blocks are reused with two consecutive MRs. In this case, we can assume that all UEs in wagon $i$ are protected from the co-channel interference caused by MRs in wagons $i-2$ and $i+2$ due to the wagon penetration loss and to the distance separating two wagons using the same frequency. For subcarriers allocation, we employ the Hungarian algorithm.

Each UE communicates with the eNB via one MR placed on the middle of the ceiling of each wagon. We assume that accurate channel state information can be available at the transmitters, even at high-speeds, using for example the predictor antenna method described in [29]. The resource allocation algorithms proposed in Section III are applied in both access and bakchaul links using the parameters summarized in Table I. Also, it is assumed that the de-correlated time $T_{d}=1$ ms [44] and the target data rate $\left(R_{\text {th }}^{U}\right)$ is the same for all UEs. Initially, the distance separating the eNB and the railway is equal to $d_{B}=100 \mathrm{~m}$ while the end of the train is located at the distance $d_{0}=0 \mathrm{~m}$ as shown in Fig. 1. Furthermore, we apply the proposed algorithm to the direct transmission where each UE communicates directly with the eNB without assistance from the relay.

TABLE I: Simulations parameters

\begin{tabular}{|l|l|}
\hline Parameter & Value \\
\hline \hline Train speed & $350 \mathrm{~km} / \mathrm{hr}$ \\
\hline Carrier frequency & $2.6 \mathrm{GHz}$ \\
\hline Total system bandwidth $\left(B_{T}\right)$ & $20 \mathrm{MHz}$ \\
\hline eNB and MR $i$ transmit antenna gains $\left(G_{B}, G_{R_{i}}\right)$ & $(14,0) \mathrm{dBi}$ \\
\hline MR $i$ and UE $m$ receive antenna gains $\left(G_{R_{i}}, G_{U_{m}}\right)$ & $(0,0) \mathrm{dBi}$ \\
\hline Total number of UEs $(M)$ & 250 \\
\hline Total number of UEs per wagon $\left(M_{i}\right)$ & 25 \\
\hline Transmit peak power at each $i^{t h} \mathrm{MR}\left(\bar{P}_{R_{i}}\right)$ & $20 \mathrm{dBm}$ \\
\hline Transmit peak power at the BS $\left(P_{B}\right)$ & $46 \mathrm{dBm}$ \\
\hline Shadowing standard deviation $(\sigma)$ & $8 \mathrm{~dB}$ \\
\hline Perpendicular penetration train loss $\left(W_{e}\right)$ & $18 \mathrm{~dB}$ \\
\hline Parallel penetration train loss $\left(W G_{e}\right)$ & $15 \mathrm{~dB}$ \\
\hline Average co-channel interference $(I)$ & $-110 \mathrm{dBm}$ \\
\hline Average noise power variance at room temperature $\left(N_{0}\right)$ & $-174 \mathrm{dBm}$ \\
\hline
\end{tabular}

\section{B. Performance of the Proposed Resource Allocation Schemes}

In all the following simulation results, we compare the proposed low complexity algorithm to the Hungarian method which corresponds to Steps 1 to 3 only of the proposed method given in Section III-B2 and to an iterative algorithm proposed in [21] which is based on a rate maximization approach. The latter method consists in allocating RBs to destinations such that backhaul performance is maximized by selecting the best RB for each destination iteratively (i.e., best channel gain).

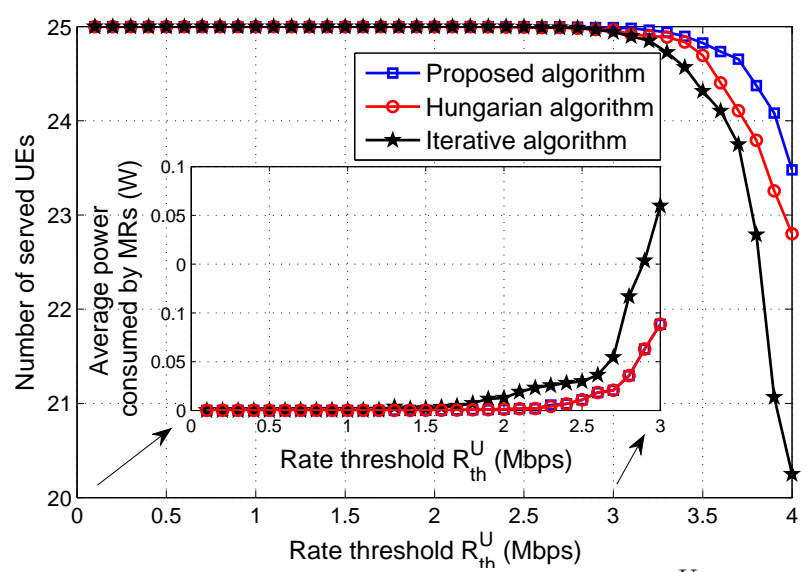

Fig. 3 Average number of served UEs versus the $R_{\text {th }}^{U}$ Mbps in the access link.

Fig. 3 plots the average number of served UEs versus the target data rate $R_{\mathrm{th}}^{U}$ for the access link (MR-UE link). It can be shown that, for all algorithms, the MRs can successfully serve the 25 UEs up to a certain $R_{\text {th }}^{U}=3 \mathrm{Mbps}$. It is noticed that the proposed and Hungarian approaches outperform the iterative algorithm in terms of power consumption. The figure shows that the whole system performance essentially depends on the backhaul link rather than the access link as the distances between transmitters and receivers are very small in this link.

In the sequel, we investigate the performance of the proposed algorithm for full system operation (i.e., backhaul and access links). Fig. 4 illustrates the total power consumption 


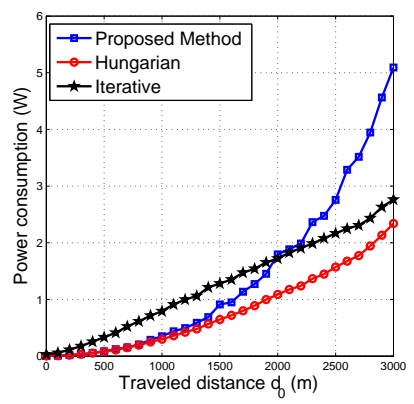

(a)

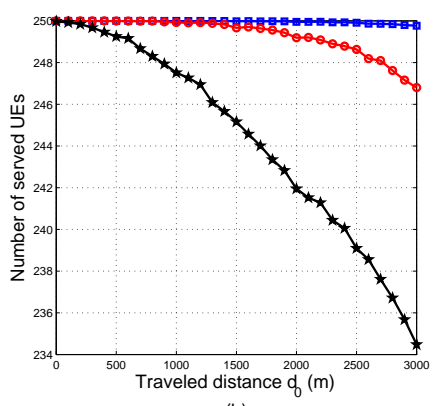

(b)
Fig. 4 Performance of the MRs versus the distance $d_{0}$ for $R_{\mathrm{th}}^{U}=0.4 \mathrm{Mbps}$.

and the number of served UEs as a function of the distance $d_{0}$ for a fixed UE target data rate $R_{\mathrm{th}}^{U}=0.4 \mathrm{Mbps}$. Note that, when all users in a wagon $i$ are successfully served, the $i^{\text {th }}$ MR target data rate becomes equal to 10 Mbps. Fig. 4 shows that the performance of our proposed algorithm outperforms the other algorithms in terms of both power consumption for traveled distance and number of served UEs. As the train moves away from the eNB, the advantages of our proposed algorithm appear clearly: the algorithm maintains the system QoS for long-range distance, e.g., for $d_{0}=2500 \mathrm{~m}$ where it still serves all the 250 UEs while the Hungarian and iterative algorithms can only serve around 248 and 240 UEs, respectively. This gain of number of served UEs is obtained at the expense of additional power consumption. Notice that for low values of traveled distance the proposed approach consumes the same amount of power as the Hungarian method (Step 1 to Step 3 of Algorithm 1). But, as the distance increases the proposed approach consumes more power to maintain the required QoS. This shows the impact of Step 4 and Step 5 that are added to efficiently manage the available power budget and meet the QoS. Compared to the sum-rate maximization approach, i.e., the iterative one, we deduce that an important gap in terms of both power consumption and number of served UEs is reached. In fact, the iterative approach allows the renouncement of serving some of its users in order to maximize the total sumrate by serving UEs with good channel conditions.

Fig. 5 depicts the impact of the $R_{\mathrm{th}}^{U}$ on the number of served UEs for fixed values $d_{0}=\{100,2000\} \mathrm{m}$. It is noticed that all algorithms can serve all UEs up to a certain $R_{\mathrm{th}}^{U^{*}}$. For instance, for $d_{0}=100 \mathrm{~m}, R_{\mathrm{th}}^{U^{*}}=\{0.8,0.7,0.5\}$ Mbps using the proposed approach, Hungarian method and iterative algorithm, respectively. This can be justified by the fact that the power budget is only enough for low $R_{\mathrm{th}}^{U}$. As the required $R_{\mathrm{th}}^{U}$ increases, the benefit of using our proposed algorithm is noticed. For instance, for $R_{\mathrm{th}}^{U}=0.6 \mathrm{Mbps}$ and $d_{0}=2000 \mathrm{~m}$, the proposed algorithm can serve around 240 UEs while the other algorithms can only serve 190 and 175 UEs as shown in Fig. 5(b). On the other hand, it is clear that the relay transmission (solid lines) outperforms the direct transmission (dashed lines). We notice the importance of the introduction of relays at the top of the train. Relays can not only help in coping with the PL increase but also in serving more UEs as they offer more available RBs. Indeed, with direct

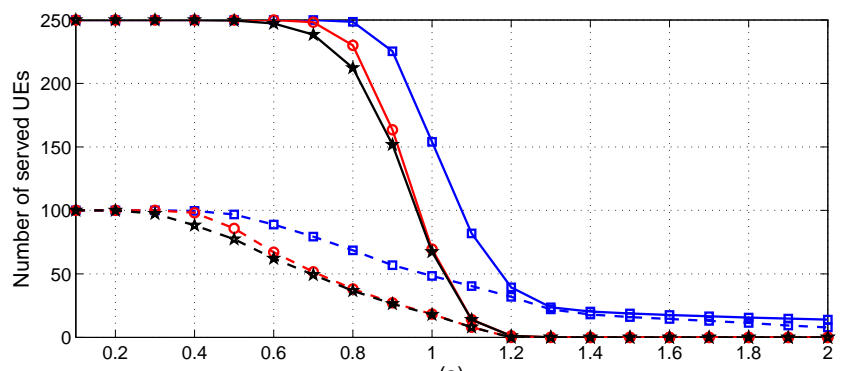

(a)

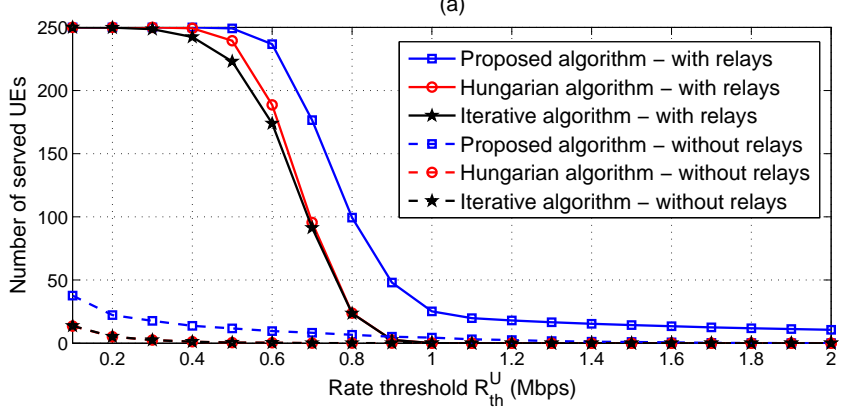

(b)

Fig. 5 Total number of served UEs versus $R_{\mathrm{th}}^{U}$ for (a) distance $d_{0}=100 \mathrm{~m}$, (b) distance $d_{0}=2000 \mathrm{~m}$.
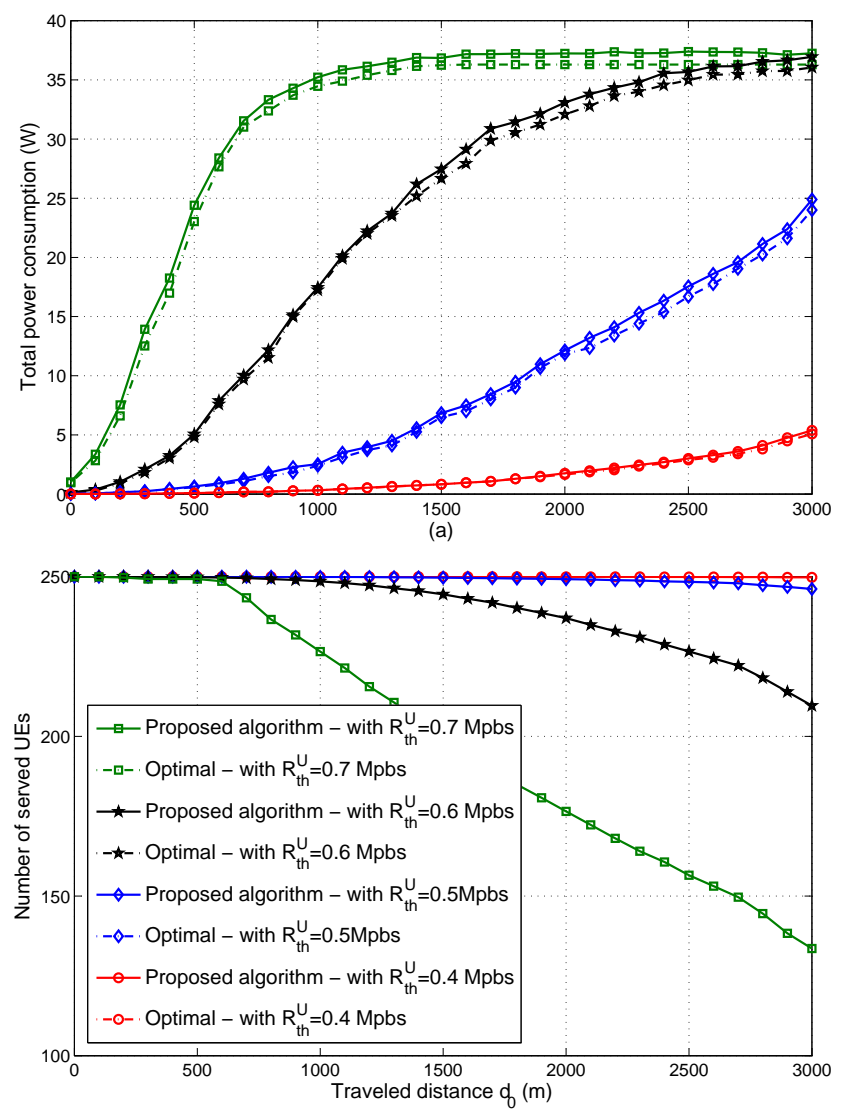

(b)

Fig. 6 Performance of the proposed algorithms for the relay transmission versus the distance $d_{0}$ for different values of $R_{\mathrm{th}}^{U}$, (a) Total power consumed by the eNB, (b) Total number of served UEs.

transmission and with $B_{T}=20 \mathrm{MHz}$, the proposed algorithms can at maximum serve 100 UEs while thanks to the relays, 
the eNB is able to communicate with 250 UEs. However, a significant decrease of the slope of the number of served UEs with the relay transmission compared to the direct transmission is obtained. Indeed, if one relay is not served, this means that the 25 UEs in the corresponding wagon are not served even after employing Step 5 of the proposed approach while with the direct transmission the increase of the target data rate per UE eliminates UE by UE. The increase of the distance $d_{0}$ implies a decrease of the system performance, for instance with $R_{\text {th }}^{U}=0.8 \mathrm{Mbps}$ and using the proposed algorithm, the number of served UEs goes from 250 with $d_{0}=100 \mathrm{~m}$ to 100 with $d_{0}=2000 \mathrm{~m}$. This means that starting from a certain distance, a handover to another closer eNB becomes a necessity to maintain the required QoS.

In Fig. 6(a), we show that the proposed method (solid lines) consumes a total power almost similar to the optimal power obtained using the dual decomposition method (dashed lines) given in Section III-B1. Also, in Fig. 6(b), we plot the corresponding number of served UEs as a function of the distance $d_{0}$ for different values of $R_{\mathrm{th}}^{U}=\{0.4,0.5,0.6,0.7\}$ Mbps. We notice that power consumption directly depends on the target data rate and increases with the distance.

\section{Complexity Analysis of the Proposed Resource Allocation Schemes}

In order to compare the complexity of the proposed and the optimal methods, we suppose hereafter that we work in the backhaul level. Note that the same analysis can be performed for the access link. For the low complexity algorithm presented in Algorithm 1 while using the Hungarian method, in the worst case scenario, the maximum number of iterations that can be reached is $L$ assuming that at each iteration only one pair of user and RB is allocated (line 4). Note that, in general, we can have multiple relay-RB associations per one iteration and thus the size of the resource allocation matrix $\epsilon$ is reduced every iteration as indicated in line 6. Therefore, the Hungarian algorithm complexity as well as the number of operations needed to compute the power expression given in (24) depend on the size of $\epsilon$ which is decreasing as the relay-RB associations are made. Thus, the complexity of the low complexity algorithm is of the order of $\sum_{i=1}^{L}((L-i)(K-i))^{3}+(L-i)(K-i)$ operations for the worst case scenario. Concerning the dual decomposition method given in Section III-B1, for a given maximum number of iterations of the subgradient method $I_{\max }$, the algorithm is executed for $I=\min \left(I_{\max }, 1 / \varepsilon^{2}\right)$ iterations where $\varepsilon$ represents the accuracy-guarantee which is defined by the difference between the best value and the iterate value [39]. We can easily see that the number of operations at each iteration is of the order of $\approx(K L)^{3}+13 K L$ operations where $(K L)^{3}$ is the number of operations for the Hungarian method and $\approx 13 K L$ is the number of operations for expressions (20), (22), and (23). Thus, the total complexity of the dual decomposition is $\approx I \times\left((K L)^{3}+13 K L\right)$. Hence, we deduce the considerable gain in terms of number of operations provided by the low complexity approach since the allocation matrix size is decreased every iteration unlike the dual composition method, where at each iteration of the subgradient method, the same matrix size is considered.

For illustrative purpose only, we present, in Table II, a comparison between the proposed algorithm and the optimal algorithm based on the dual decomposition in terms of CPU time and the number of operations. For 100 realizations, results show that the optimal method requires, on average, more time to converge. We can clearly see that the computational complexity is considerably reduced using the proposed low complexity algorithm due to the dynamic size reduction performed at each iteration. Recall that the latter algorithm achieves close performance to the optimal solution as it is shown in Fig. 6. All tests were performed on a desktop machine featuring an Intel Xeon CPU and running Windows 7 Professional. The clock of the machine is set to $2: 66 \mathrm{GHz}$ with a 48 GB memory.

TABLE II: Complexity of the Algorithms

\begin{tabular}{|l||c|c|}
\hline & Dual Decomposition & Proposed algorithm \\
\hline \hline CPU times $(L=4)$ & 0.0885 & 0.0005 \\
\hline Operations $(L=4)$ & $9 \times 10^{4}$ & $8 \times 10^{2}$ \\
\hline CPU times $(L=10)$ & 0.1172 & 0.0017 \\
\hline Operations $(L=10)$ & $2.1 \times 10^{7}$ & $9.8 \times 10^{5}$ \\
\hline
\end{tabular}

\section{Performance of the Proposed Planning Method}

Next, we present the performance of the BS planning for high-speed train with moving relays studied in Section III-C.

In Fig. 7, we plot the optimum distance as a function of the number of relays and for different $R_{\text {th }}^{U}$ values. Note that the total shared data rate by one relay is given by $25 R_{\text {th }}^{U}$ (For $R_{\mathrm{th}}^{U}=0.144 \mathrm{Mbps}$, the target rate per relay is $3.6 \mathrm{Mbps}$, thus per train is $3.6 L \mathrm{Mbps})$. As it is expected, for a fixed $R_{t h}^{U}$ value, the optimum distance separating the BSs is inversely proportional to the number of relays because when the latter increases more power is needed to maintain the desired QoS. We also notice that we can increase the distance by setting low values of $R_{\text {th }}^{U}$, i.e., low QoS. Also, in this figure, we compare the performance of our proposed approach (denoted by "indep. MRs") to those of the ideal case where relays cooperate together to serve users, denoted by "Coop. MRs". In this scenario, relays are assumed to be connected to each other via an optical cable that allows users belonging to wagon $i$ to be served via a relay placed on the top of wagon $j \neq i$. Although this scenario requires a more developed problem formulation and a more sophisticated algorithm, we present in Fig. 7 a suboptimal solution based on a meta-heuristic algorithm that optimizes the inter-BS distance in addition to the number of users associated to each relay. We notice that the proposed scheme closely achieves the inter-cell distance of the ideal case: a difference of 200-300 meters. This can be explained by the fact that at cell edge, the BS uses its full power. Hence, the coop. case will need to reduce the number of users of the wagons in outage, in order to serve them by other relays closer to the BS. This will lead to an increase of the power consumption allocated to the supporting relays which does not offer large flexibility to the system. Note that 


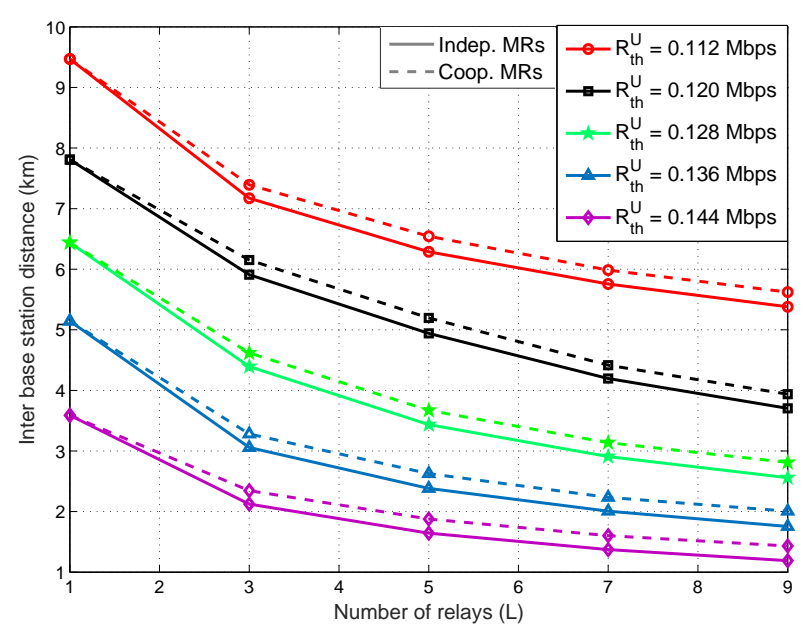

Fig. 7 Planning for different values of $R_{\mathrm{th}}^{U}$.

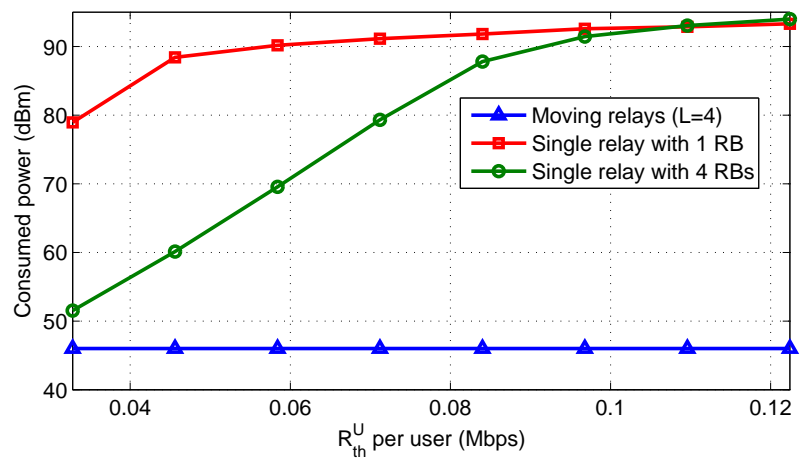

Fig. 8 Comparison between the single relay and the moving relays scenarios using the proposed planning approach.

this increase follows an exponential function equivalent to $A_{\text {th }}$ given in (25).

Fig. 8 shows the advantage of using moving relays over the traditional single relay scheme adopted in [15]. In fact, for the same total number of users, we plot the total consumed power as a function of $R_{\text {th }}^{U}$ for both single and 4 MRs networks by adopting the optimal inter-BS distance obtained for the $4 \mathrm{MRs}$ case. We can clearly see that by using MRs, we do not exceed the total power budget fixed at $46 \mathrm{dBm}$ while the single relay scheme requires an enormous power consumption even if it is using 4 RBs for the transmission. Hence, in contrast with our proposed scheme, the single relay scheme cannot be applied in practical scenario with high rates.

In Fig. 9, we test the LTE planning scheme in two different scenarios with $L=5$ and $L=7$ relays with the presence of fading and shadowing effects. Each network is composed of 3 eNBs placed at $0 \mathrm{~m}, d_{\mathrm{BS}}^{*} \mathrm{~m}$ and $2 d_{\mathrm{BS}}^{*} \mathrm{~m}$ and 25 users per wagon with $R_{\mathrm{th}}^{U}=0.128 \mathrm{Mbps}$. The optimal inter-BS distances are presented in Table III in $\mathrm{km}$. For each network, we apply the proposed LTE planning and plot the percentage of non-served users as a function of the train position after averaging over 1000 independent Monte Carlo runs. We clearly notice that the percentage of non-served users is less than $0.5 \%$ in the zone covered by the eNBs. We can also see that the percentage of non-served users decreases in the case of overlap $(\tau=$

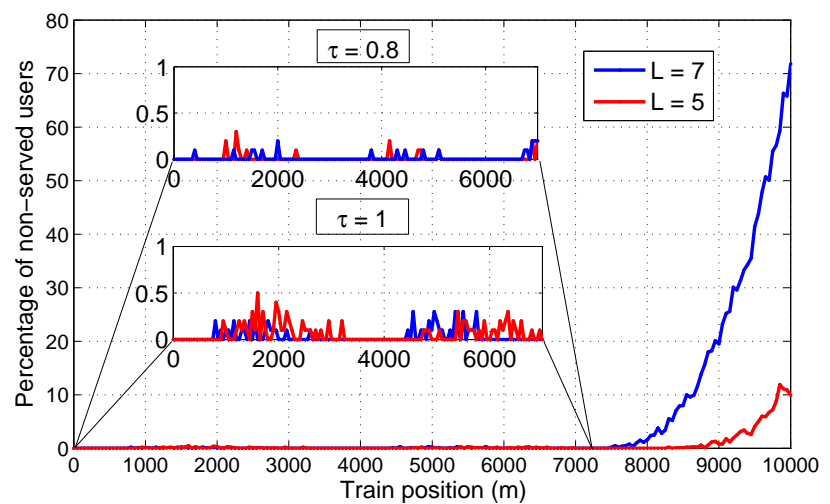

Fig. 9 Percentage of non-served users with and without overlapping.

$0.8)$ compared with the non-overlap case $(\tau=1)$. Indeed, the introduced tolerance provides more reliability to the railway communication and reduces the risk of handover failure.

TABLE III: Optimal inter-BS distances in $\mathrm{km}$

\begin{tabular}{|l||l|l|}
\hline & $\tau=1$ & $\tau=0.8$ \\
\hline$L=5$ & 3.8 & 3.4 \\
\hline$L=7$ & 3.2 & 2.8 \\
\hline
\end{tabular}

\section{CONCLUSION}

This paper studied the problem of resource allocation for high-speed train equipped with multiple moving relays in OFDMA systems. The objective is to minimize the total transmit power consumption while serving the passengers by meeting their requested throughput. Optimal solution is derived using the optimal decomposition method in order to optimize both power and subcarriers allocation. Moreover, we proposed a practical algorithm to find a suboptimal resource allocation solution. Interestingly, it has been shown that the performance of the proposed scheme using moving relays achieves close performance to those of the dual decomposition method and outperforms the performance of some previously proposed algorithms as well as the direct transmission. We also proposed a planning method to optimally determine the inter-base station distance according to given quality of service parameters. Its efficiency is verified using Monte Carlo simulations. Finally, simulation results show that our proposed algorithm maintains the system quality-of-service even for long-range distance with near optimal power consumption.

The investigation of the cooperation among moving relays for high-speed train communications represents an interesting extension of this work where the objective is to develop optimized mechanisms in order to enhance the system performance and ensure seamless handover with larger inter-base station distances. In addition to the power and subcarriers allocation, another challenging dimension can be introduced to the framework: the user-relay association.

\section{REFERENCES}

[1] B. Ai, X. Cheng, T. Kurner, Z.-D. Zhong, K. Guan, R.-S. He, L. Xiong, D. Matolak, D. Michelson, and C. Briso-Rodriguez, "Challenges toward wireless communications for high-speed railway," IEEE Transactions on Intelligent Transportation Systems, vol. 15, no. 5, pp. 2143-2158, Oct. 2014. 
[2] Y. Dong, P. Fan, and K. Ben Letaief, "High-speed railway wireless communications: Efficiency versus fairness," IEEE Transactions on Vehicular Technology, vol. 63, no. 2, pp. 925-930, Feb. 2014.

[3] J. Wang, H. Zhu, and N. Gomes, "Distributed antenna systems for mobile communications in high speed trains," IEEE Journal on Selected Areas in Communications, vol. 30, no. 4, pp. 675-683, May 2012.

[4] J. Calle-Sanchez, M. Molina-Garcia, J. I. Alonso, and A. FernandezDuran, "Long term evolution in high speed railway environments: Feasibility and challenges," Bell Labs Technical Journal, vol. 18, no. 2 , pp. 237-253, Sep. 2013.

[5] 3rd Generation Partnership Project, "Technical specification group radio access network; Mobile relay for E-UTRA," 3GPP TR 36.836 V 10.0.0, Release 10, Jun. 2012.

[6] A. Diaz Zayas, C. Garcia Perez, and P. Merino Gomez, "Thirdgeneration partnership project standards: For delivery of critical communications for railways," IEEE Vehicular Technology Magazine, vol. 9, no. 2, pp. 58-68, Jun. 2014.

[7] A. Sniady and J. Soler, "LTE for railways: Impact on performance of ETCS railway signaling," IEEE Vehicular Technology Magazine, vol. 9, no. 2 , pp. 69-77, June 2014.

[8] 3rd Generation Partnership Project (3GPP), "3GPP TR 36.836 3GPP TSG RAN study on mobile relay for evolved universal terrestrial radio access E-UTRA," V 2.0.2, Release 12, Jul. 2013.

[9] Y. Sui, A. Papadogiannis, W. Yang, and T. Svensson, "Performance comparison of fixed and moving relays under co-channel interference," in IEEE Globecom Workshops (GC Workshops 2012), Anaheim, CA USA, Dec. 2012.

[10] Y. Sui, A. Papadogiannis, and T. Svensson, "The potential of moving relays - A performance analysis," in Proc. of the 75th IEEE Vehicular Technology Conference (VTC Spring'2012), Yokohama, Japan, May 2012.

[11] Y. Sui, Z. Ren, W. Sun, T. Svensson, and P. Fertl, "Performance study of fixed and moving relays for vehicular users with multi-cell handover under co-channel interference," in International Conference on onnected Vehicles and Expo (ICCVE 2013), Las Vegas, NV, USA, Dec. 2013.

[12] W. Li, C. Zhang, X. Duan, S. Jia, Y. Liu, and L. Zhang, "Performance evaluation and analysis on group mobility of mobile relay for LTE advanced system," in Proc. of the 76th IEEE Vehicular Technology Conference (VTC Fall'2012), Quebec, Canada, Sept. 2012.

[13] S. Scott, J. Leinonen, P. Pirinen, J. Vihriälä, V. Phan, and M. Latva-aho, "A cooperative moving relay node system deployment in a high speed train," in Proc. of the 77th IEEE Vehicular Technology Conference (VTC Spring'2013), Dresden, Germany, Jun. 2013.

[14] A. Alsharoa, H. Ghazzai, E. Yaacoub, and M.-S. Alouini, "Energyefficient two-hop LTE resource allocation in high speed trains with moving relays," in in Proc. of the 12th International Symposium on Modeling and Optimization in Mobile, Ad Hoc, and Wireless Networks (WiOpt 2014), Hammamet, Tunisia, May 2014.

[15] S. Xu, G. Zhu, C. Shen, Y. Lei, and Z. Zhong, "Utility-based resource allocation in high-speed railway wireless networks," EURASIP Journal on Wireless Communications and Networking, vol. 68, no. 1, 2014.

[16] C. Zhang, P. Fan, K. Xiong, and P. Fan, "Optimal power allocation with delay constraint for signal transmission from moving train to base stations in high-speed railway scenarios," IEEE Transactions on Vehicular Technology, vol. 64, no. 12, pp. 5775-5788, Dec. 2015.

[17] Y. Zhao, X. Li, X. Zhang, Y. Li, and H. Ji, "Multidimensional resource allocation strategy for high-speed railway MIMO-OFDM system," in IEEE Global Communications Conference (GLOBECOM 2012), Anaheim, CA, USA, Dec. 2012.

[18] H. Zhu, "Radio resource allocation for OFDMA systems in high speed environments," IEEE Journal on Selected Areas in Communications, vol. 30, no. 4, pp. 748-759, May 2012.

[19] K. X. Chuang Zhang, Pingyi Fan, "Downlink resource allocation for the high-speed train and local users in OFDMA systems," in IEEE Wireless Communications and Networking Conference (WCNC 2015), New Orleans, LA, USA, Mar. 2015.

[20] T. Riihonen, R. Wichman, and S. Werner, "Evaluation of OFDM(A) relaying protocols: Capacity analysis in infrastructure framework," IEEE Transactions on Vehicular Technology, vol. 61, no. 1, pp. 360-374, Jan. 2012.

[21] R. Atat, E. Yaacoub, M. Alouini, and A. Abu-Dayya, "Heterogeneous LTE/802.11a mobile relays for data rate enhancement and energyefficiency in high speed trains," in Proc. of the IEEE Globecom Workshops (GC Workshps'2012), Anaheim, CA, USA, Dec. 2012.

[22] Z. Liu and P. Fan, "An effective handover scheme based on antenna selection in ground-train distributed antenna systems," IEEE Transactions on Vehicular Technology, vol. 63, no. 7, pp. 3342-3350, Sept. 2014.
[23] Y.-B. Lin, S.-N. Yang, and C.-T. Wu, "Improving handover and drop-off performance on high-speed trains with multi-RAT," IEEE Transactions on Intelligent Transportation Systems, vol. 15, no. 6, pp. 2720-2725, Dec. 2014.

[24] M. Pan, T. Lin, and W. Chen, "An enhanced handover scheme for mobile relays in LTE-A high-speed rail networks," IEEE Transactions on Vehicular Technology, vol. 64, no. 2, pp. 743-756, Feb. 2015.

[25] P. Kyösti, J. Meinilä, L. Hentilä, X. Zhao, T. Jämsä, C. Schneider, M. Narandzić, M. Milojević, A. Hong, J. Ylitalo, V.-M. Holappa, M. Alatossava, R. Bultitude, Y. de Jong, and T. Rautiainen, "WINNER II Channel Models," Tech. Rep., [Available Online: http://www.istwinner.org.html], Sept. 2007.

[26] Z. Wang, E. Tameh, and A. Nix, "Joint shadowing process in urban peerto-peer radio channels," IEEE Transactions on Vehicular Technology, vol. 57, no. 1, pp. 52-64, Jan. 2008.

[27] K. Yamamoto, A. Kusuda, and S. Yoshida, "Impact of shadowing correlation on coverage of multihop cellular systems," in Proc. of the IEEE International Conference on Communications (ICC'2006), Istanbul, Turkey, Jun. 2006

[28] ETSI TR 101112 (v3.2.0), "Universal mobile telecommunications system (UMTS); Selection procedures for the choice of radio transmission technologies of the UMTS (UMTS 30.03)," Tech. Rep., [Available Online: http://www.etsi.org/deliver/], Apr. 1998.

[29] M. Sternad, M. Grieger, R. Apelfrojd, T. Svensson, D. Aronsson, and A. Martinez, "Using predictor antennas for long-range prediction of fast fading for moving relays," in Proc. of the IEEE Wireless Communications and Networking Conference Workshops (WCNCW'2012), Paris, France, Apr. 2012.

[30] N. Jamaly, R. Apelfrojd, A. Martinez, M. Grieger, T. Svensson, M. Sternad, and G. Fettweis, "Analysis and measurement of multiple antenna systems for fading channel prediction in moving relays," in 8th European Conference on Antennas and Propagation (EuCAP 2014), April The Hague, the Netherlands, Apr. 2014

[31] D.-T. Phan-Huy, M. Sternad, and T. Svensson, "Making 5G adaptive antennas work for very fast moving vehicles," IEEE Intelligent Transportation Systems Magazine, vol. 7, no. 2, pp. 71-84, Summer, 2015.

[32] L. Liu, C. Tao, J. Qiu, and H. Chen, "An performance study for sectorised antenna based Doppler diversity in high-speed railway communications," Radio engineering, vol. 20, no. 4, pp. 866-871, Dec. 2011.

[33] J. Li and Y. Zhao, "Radio environment map-based cognitive Doppler spread compensation algorithms for high-speed rail broadband mobile communications," EURASIP Journal on Wireless Communications and Networking, vol. 2012, Aug. 2012.

[34] Y. Zhou, Z. Hou, Z. Pan, J. Shi, and J. Wang, "Dynamic Doppler tracking for LTE-based broadband communications on high speed rails," in Proc. of the IEEE China Summit International Conference on Signal and Information Processing (ChinaSIP 2013), Beijing, China, Jul. 2013.

[35] W. Yu and R. Lui, "Dual methods for nonconvex spectrum optimization of multicarrier systems," IEEE Transactions on Communications, vol. 54, no. 7, pp. 1310-1322, Jul. 2006.

[36] S. Boyd and A. Mutapcic, "Stochastic Subgradient Methods," Notes for EE364, Stanford University, Winter. 2006-07.

[37] R. Rardin, Optimization in Operations Research. Upper Saddle River, NJ: Prentice Hall, 1998.

[38] H. W. Kuhn, "The Hungarian Method for the Assignment Problem," 50 Years of Integer Programming 1958-2008. Berlin, Heidelberg. ch. 2, pp. 29-47, 2010.

[39] S. Boyd and L. Vandenberghe, Convex Optimization. New York, NY, USA: Cambridge University Press, 2004.

[40] H.Holma and A.Toskala, LTE for UMTS: OFDMA and SC-FDMA Based Radio Access. John Wiley \& Sons, 2009.

[41] A. Nguyen, Y. Huang, and B. Rao, "Novel partial feedback schemes and their evaluation in an OFDMA system with CDF based scheduling," in Asilomar Conference on Signals, Systems and Computers, Pacific Grove, CA, USA, Nov. 2013

[42] 3rd Generation Partnership Project (3GPP), "Evolved universal terrestrial radio access E-UTRA; Physical channels and modulation," 3GPP TS 36.211 3GPP V 11.2.0, Release 11.

[43] — "Evolved universal terrestrial radio access E-UTRA; Physical layer procedures," 3GPP TS 36.213 3GPP V 11.2.0, Release 11, Apr 2013.

[44] G. Marfia, M. Roccetti, A. Amoroso, and G. Pau, "Safe driving in LA: Report from the greatest intervehicular accident detection test ever," IEEE Transactions on Vehicular Technology, vol. 62, no. 2, pp. 522-535, Feb. 2013 


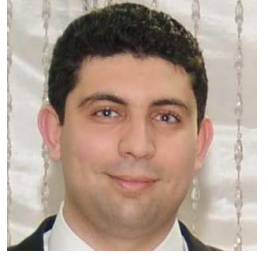

Hakim Ghazzai (S'12, M'15) was born in Sousse, Tunisia. He is currently working as a research scientist at Qatar Mobility Innovations Center (QMIC) Doha, Qatar. He received his $\mathrm{PhD}$ degree in Electrical Engineering from King Abdullah University of Science and Technology (KAUST), Saudi Arabia in 2015. He received his Diplome d'Ingenieur in telecommunication engineering and Master of Science degree in high-rate transmissions systems from the Ecole Superieure des Communications de Tunis (SUP'COM), Tunis, Tunisia in 2010 and 2011, respectively. His general research interests includes mobile and wireless networks, green communications, internet of things, and optimization.

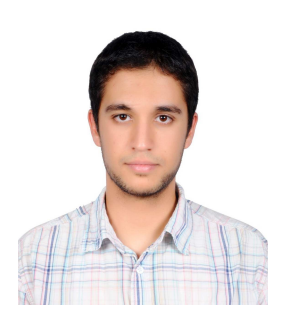

Taha Bouchoucha (S'14) received his Diplme dIngnieur degree from the Ecole Polytechnique de Tunisie (EPT), Tunisia, in 2013, and his M.S. degree in electrical engineering from King Abdullah University of Science and Technology (KAUST), KSA, in 2015. Currently, he is pursuing his Ph.D. degree in electrical engineering at the University of California Davis (UC Davis). His research interests include beamforming in MIMO radar systems and dynamic spectrum allocation in HCRAN.

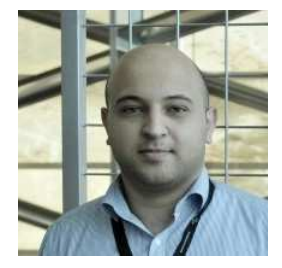

Ahmad Alsharoa (S'12) was born in Irbid, Jordan. He received the B.Sc degree (with honors) from Jordan University of Science and Technology (JUST), Irbid, Jordan, in 2011 and the M.Sc. degree from King Abdullah University of Science and Technology (KAUST), Thuwal, Saudi Arabia, in 2013 both in Electrical Engineering. He is currently pursuing the Ph.D. degree in the Electrical and Computer Engineering at Iowa State University (ISU), Ames, Iowa, USA. His current research interests include: energy harvesting, cooperative relay networks, MIMO communications, energy efficient power allocation, and cognitive radio networks.

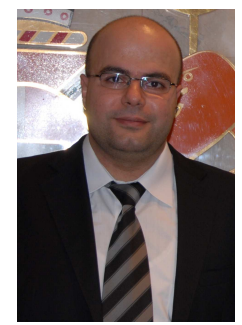

Elias Yaacoub (S'07, M'10, SM'14) received the B.E. degree in Electrical Engineering from the Lebanese University in 2002, the M.E. degree in Computer and Communications Engineering from the American University of Beirut (AUB) in 2005, and the $\mathrm{PhD}$ degree in Electrical and Computer Engineering from AUB in 2010. He worked as a Research Assistant in the American University of Beirut from 2004 to 2005, and in the Munich University of Technology in Spring 2005. From 2005 to 2007 , he worked as a Telecommunications Engineer with Dar Al-Handasah, Shair and Partners. From November 2010 till December 2014, he worked as a Research Scientist / R\&D Expert at the Qatar Mobility Innovations Center (QMIC). Afterwards, he joined Strategic Decisions Group (SDG) where he worked as a Consultant till February 2016. $\mathrm{He}$ is currently an Associate Professor at the Arab Open University (AOU). His research interests include Wireless Communications, Resource Allocation in Wireless Networks, Intercell Interference Mitigation Techniques, Antenna Theory, Sensor Networks, and Bioinformatics.

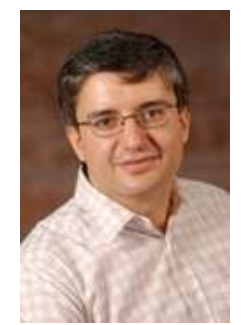

Mohamed-Slim Alouini (S'94, M'98, SM'03, F'09) was born in Tunis, Tunisia. He received the Ph.D. degree in Electrical Engineering from the California Institute of Technology (Caltech), Pasadena, CA, USA, in 1998. He served as a faculty member in the University of Minnesota, Minneapolis, MN, USA, then in the Texas A\&M University at Qatar, Education City, Doha, Qatar before joining King Abdullah University of Science and Technology (KAUST), Thuwal, Makkah Province, Saudi Arabia as a Professor of Electrical Engineering in 2009. His current research interests include the modeling, design, and performance analysis of wireless communication systems.

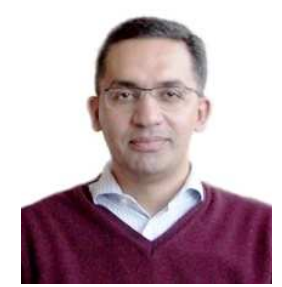

Tareq Al-Naffouri (M'10) received the B.S. degrees in mathematics and electrical engineering (with first honors) from King Fahd University of Petroleum and Minerals, Dhahran, Saudi Arabia, in 1994, the M.S. degree in electrical engineering from the Georgia Institute of Technology, Atlanta, in 1998, and the $\mathrm{Ph} . \mathrm{D}$. degree in electrical engineering from Stanford University, Stanford, CA, in 2004.

$\mathrm{He}$ was a visiting scholar at California Institute of Technology, Pasadena, CA, from January to August 2005 and during summer 2006. He was a Fulbright scholar at the University of Southern California from February to September 2008. He has held internship positions at NEC Research Labs, Tokyo, Japan, in 1998, Adaptive Systems Lab, University of California at Los Angeles in 1999, National Semiconductor, Santa Clara, CA, in 2001 and 2002, and Beceem Communications Santa Clara, CA, in 2004. He is currently an Associate at the Electrical Engineering Department, King Abdullah University of Science and Technology (KAUST). His research interests lie in the areas of adaptive and statistical signal processing and their applications to wireless communications, seismic signal processing, and in multiuser information theory. He has recently been interested in compressive sensing and random matrix theory and their applications. He has over 130 publications in journal and conference proceedings, 9 standard contributions, 10 issued patents, and 4 pending.

Dr. Al-Naffouri is the recipient of the IEEE Education Society Chapter Achievement Award in 2008 and Al-Marai Award for innovative research in communication in 2009. Dr. Al-Naffouri has also been serving as an Associate Editor of Transactions on Signal Processing since August 2013. 